rupture of the spleen and, less commonly, the liver. Bruising of the anterior abdominal wall is surprisingly uncommon in association with major intra-abdominal damage, probably because many such cases follow trauma to a relaxed and unguarded abdominal wall, so that the brunt of the injury falls upon the abdominal contents rather than the wall itself. Rupture of a solid organ is suggested by the signs of blood loss together with abdominal guarding and tenderness, with dullness in one or both flanks. A rigid, silent abdomen, without evidence of haemorrhage, is more in favour of rupture of a hollow viscus. Loin tenderness and bruising, together with haematuria, indicate renal damage.

Diagnosis is testing enough in uncomplicated cases, but when the patient has other injuries, and in particular when he is unconscious from a head injury, the problem becomes still more puzzling. Naturally the surgeon looks to ancillary aids. Plain $x$-rays of the abdomen are of limited value, though identification of free gas naturally confirms rupture of a hollow viscus. An emergency intravenous pyelogram is valuable when renal injury is suspected.

Diagnostic paracentesis of the abdominal cavity has been popular for many years in the U.S.A. in cases both of abdominal trauma and of acute abdominal emergency ${ }^{2}$ but has been much less practised in Great Britain. However, W. N. W. Baker and his colleagues ${ }^{3}$ reported a series of 101 acute abdominal emergencies seen at the Central Middlesex Hospital. Paracentesis was positive in about $80 \%$ of their cases of intraperitoneal haemorrhage and perforation, though less than $10 \%$ of localized inflammatory disease gave a positive finding. In cases of blunt abdominal trauma many reports from the United States of tapping two or four quadrants of the peritoneal cavity have shown that, where blood is obtained, $80 \%$ or more of the patients are found to have intra-abdominal injury at laparotomy. But unfortunately there is a high percentage of false negative results.

In an attempt to refine this diagnostic aid the technique of peritoneal lavage was introduced by H. D. Root and his colleagues in 1965.4 E. R. Thal and G. T. Shires 5 now report their experience of 267 patients undergoing peritoneal lavage after abdominal trauma in Dallas, Texas. The technique used was to insert a peritoneal dialysis catheter via a trocar in the lower midline between the umbilicus and the pubis. The catheter was aspirated, and if non-clotting blood returned freely the diagnosis of haemoperitoneum was confirmed and the patient was taken to the operating theatre. If no aspirate was obtained, a litre of Ringer's solution was infused for 5-10 minutes and then siphoned out of the peritoneal cavity. The fluid was examined for blood, amylase, bile, and bacteria. The procedure was not carried out if the diagnosis was obvious clinically, but if a diagnosis could not be made a two-quadrant needle paracentesis was performed. Lavage was carried out only if this procedure in turn was negative. Lavage was also performed when the diagnosis was complicated by a head injury or an altered state of consciousness produced by alcohol, shock, or drugs. Peritoneal lavage was contraindicated in patients who had undergone multiple abdominal operations and in whom the presence of adhesions increased the risk of penetrating the gut. Pregnancy, gross abdominal distension, and gunshot or stab wounds were further contraindications. The criteria for positive results on lavage were considered to be the presence of gross non-clotted blood or the presence of more than 100,000 red cells or 500 white cells per cubic millimetre, a raised amylase level, or the presence of bile or bacteria. Any of these factors was considered to be an indication for laparotomy.

Many patients were spared exploratory laparotomy on the basis of negative lavage results, and only two patients subsequently needed surgery who were admitted to hospital and initially observed. These authors reported 173 patients $(64.8 \%)$ as having negative results from lavage. Among them were nine false negative results $(3 \cdot 1 \%)$. There were also ten false positive results $(3.5 \%)$. But the procedure appears to be a valuable aid whether its results are negative or positive. However, it is not free from complications, and indeed these occurred in 13 patient $(4.5 \%)$. They included infusion of the lavage fluid into the abdominal wall, penetration of the bladder or large small bowel, and laceration of a mesenteric vessel and retroperitoneal haemorrhage. Moreover, lavage is very inaccurate in the diagnosis of retroperitoneal injuries, and most pancreatic trauma was not detected by this technique. An increase of amylase more generally indicates laceration of the bowel than pancreatic trauma.

It should be emphasized that these authors suggest peritoneal lavage only as an aid when the diagnosis is in doubt. Laparotomy should not be delayed when visceral rupture or intraperitoneal bleeding is clinically obvious.

1 Williams, R. D., and Yurko, A. A., American Fournal of Surgery, $1966,111,477$

2 Newhof. H., and Cohen, I., Annals of Surgery, 1926, 83, 454.

3 Baker. W. N. W. Mackie, D. B., and Newcombe, J. F. British Medical fournal, 1967, 3, 146

Root, H. D. Hauser, C .W., McKinley, C. R., LaFave, J. W., and Mendiola, R. P., Surgery, 1965, 57, 633.

5 Thal. E. R., and Shires, G. T., American fournal of Surgery, 1973, 125, 64 .

\section{Syndrome of Gilles de la Tourette}

The pages of the history of medicine contain many personalities who have given their names to syndromes or diseases, and neurology has many examples. Few more colourful names will be found than that of G. Gilles de la Tourette, ${ }^{1}$ who in 1885 described nine patients with persistent multiple tics beginning in childhood, five of whom had verbal tics consisting of repeated expostulations of obscenities and swear-words. To this antisocial behaviour is given the term coprolalia. Other features of the syndrome which have been recognized include the tendency of the patient to repeat words or phrases spoken to him (echolalia), and to show compulsive imitation of movements or gestures (echokinesis or echopraxia). The compulsive, multiple, repetitive, complex movements (tics), particularly of the face and arms, gave rise to the term "maladie des tics convulsifs" or "impulsifs".2

The vocal tics show all varieties from grunts or barks to fully formed and explicit obscenities. Coprolalia is common in chronic paranoid schizophrenia and, some would say, too common as a social "grace" in a proportion of the normal male population. Tics similarly are not uncommon, and there is a suggestion that the syndrome of Gilles de la Tourette is simply the extreme end of the spectrum of tics in gentral. ${ }^{3}$ However, the fully fledged picture of the syndrome is striking and relatively rare.

The condition begins in childhood, in $85 \%$ before the age of 11 years. Males are affected three times as commonly as 
females. ${ }^{4}$ Though S. A. K. Wilson's patient ${ }^{2}$ became insane, leading him to suggest that the prognosis was sinister, many patients remain static in their condition or improve over the years. ${ }^{3} 5$ The effects of various remedies have been tried and haloperidol in high dosage has been advised for suppressing the socially troublesome symptoms. ${ }^{6}$

The condition has always been classed as a psychiatric disease, and the increased frequency of left-handedness, emotional trauma in childhood, and a family history of mental disease have supported this. There is almost no neuroanatomical information about the syndrome because it is not fatal, and the two reports which are available do not disclose striking abnormalities. ${ }^{78}$ The electroencephalogram may be abnormal in up to $75 \%$ of cases, ${ }^{45}$ though it is generally believed that there is no neurological abnormality in these patients.

A recent paper by R. D. Sweet and his colleagues from the Cornell Medical Center, New York, ${ }^{9}$ reports the careful clinical examination of 22 patients with this syndrome. Eleven of the patients had mild motor asymmetries ranging from a decreased tone or impaired arm swing on one side to a Babinski sign. This high frequency of neurological abnormalities is striking, and it shows the value of a careful neurological examination in the investigation of patients with psychiatric disorders. However, the results must be accepted with some caution, since most of the neurological abnormalities were minor, subtle, and difficult to assess in an objective fashion.

Electroencephalographic abnormalities were found in 12 of 17 of these patients. These included paroxysmal features with bilateral sharp waves in eight, and a background disorganization with slowing in seven. Again these electroencephalographic abnormalities, though strikingly in excess of the frequency in a "normal" population, are not specific.

The authors interpret the findings as suggesting that a neurological disorder underlies Gilles de la Tourette's syndrome, though admitting that they offer no clarification of its nature. There is no reason to argue with this view, for many if not all psychiatric diseases must eventually prove to have a struotural, biochemical, or electrophysiological basis.

1 Gilles de la Tourette, G., Archives de Neurologie, 1885, 9, 19.

2 Wilson, S. A. K., Neurology, ed. A. N. Bruce, p. 1631. London, Arnold, 1940 .

3 Corbett, J. A., Mathews, A. M., Connell, P. H., and Shapiro, D. A., British fournal of Psychiatry, 1969, 115, 1229.

1 Fernando, S. J. M., British . Fournal of Psychiatry, 1967, 113, 607.

5 Field, J. R., Corbin, K. B., Goldstein, N. P., and Klass, D. W., Neurology

(Minneapolis), 1966, 16, 453 . Psvchiatry, 1964, 121, 608.

DeWulf, A., and van Bogaert, L., Monatsschrift für Psychiatrie und Neurologie, 1941, 104, 53 .

8 Balthasar, K., Archiv für Psychiatrie und Nervenkrankheiten, 1957, 195, 531.

o Sweet, R. D., Solomon, G. E., Wayne, H., Shapiro, E., and Shapiro, A. K., fournal of Neurology, Neurosurgery and Psychiatry, 1973, 36, 1.

\section{Better Aids for the Deaf}

In 1947 the Medical Research Council's report on hearing aids and audiometers ${ }^{1}$ outlined the performance characteristics of a hearing aid. Its conclusions were based on the examination of a large number of deaf people, and they were intended to give the maximum benefit to the average deaf person, whatever the type of deafness. The M.R.C.'s specifications also gave hearing aid manufacturers, who had not had the opportunity of studying large numbers of the deaf, a basis on which to design aids and resulted in a considerable improvement in the design and fitting of them.

The next great step forward came when transistors replaced valves, enabling the size of the instrument and energy requirements to be reduced. Subsequently the Medresco aid was developed further and other Medresco aids have been produced in an attempt to cater for more of the deaf population not suited by the original specifications. The time has now come when the design of hearing aids should be examined afresh.

The average cost to the Health Department of all the services required to fit and supply a free hearing aid is about $£ 10$, and the keenness, ability, and experience of the staffs of the distribution centres have resulted in a first-class service, better in fact than most commercial suppliers can give. But in contrast to commercial instruments the Medresco aid has an inferior outward design and no version for adult post-auricular wear is available. The majority of aids are issued to the elderly, who often live alone, so that careful design to make the aid easy to use is of great importance, particularly for persons with stiff fingers. The average age of persons applying for Medresco hearing aids is nearly $70 .^{2}$ But deaf persons are sensitive about being seen to be wearing an aid, and private fortunes have been amassed by taking advantage of this human weakness and of the natural suspicion of anything given freely, though a better appearance does not necessarily go with a better performance. Moreover, hearing tests give little information of the type of aid required, and the opportunity to try a series of instruments is of much more importance.

The Consumers' Association ${ }^{3}$ recently conducted a survey among 1,500 people who had purchased commercial aids. Its report emphasizes the advisability of obtaining expert medical advice before consulting a hearing aid dealer, and the Hearing Aid Industry Association's code of ethical business practice requires a dealer to ensure a person has done so before his first aid is fitted, but unfortunately this rule is frequently broken. Less than a quarter of the sample were offered a choice of aids at widely different prices, and only half the sample were allowed a period of trial with the aid. Since they had bought their aids $30 \%$ of the sample had been contacted by the dealer with the suggestion that they might require a newer aid. Of the 1,000 persons owning both a Medresco and a commercial aid half found they heard better with the Medresco, yet $60 \%$ of them preferred the commercial aid. Many preferred post-aural aids because they were less conspicuous, produced less clothing noise, and were more comfontable and simple to use.

A proportion of deaf people remain for whom the present aids are not much help and may be a positive hindrance. They usually have a considerable loss of hearing owing to defects in the inner ear. If the sounds of normal speech are magnified sufficiently to be heard, they find that somewhat louder sounds become unbearably loud. Techniques have been developed to overcome this problem, even to the extent of matching the loudness of each sound to the patient's remaining hearing, ${ }^{4}$ but though they ensure that the hearing aid is tolerable to wear and make conversation loud enough to hear they seldom improve the patient's understanding of speech.

Thus in arguments over the relative value of commercial and Medresco aids the real object of the aid is apt to be lost sight of-that is, to improve intelligibility. Plain ampli- 